\title{
HARNACK'S INEQUALITY FOR COOPERATIVE WEAKLY COUPLED ELLIPTIC SYSTEMS
}

\author{
Ari Arapostathis \\ Department of Electrical and Computer Engineering \\ The University of Texas at Austin \\ Austin, Texas 78712 \\ Mrinal K. Ghosh \\ Department of Mathematics \\ Indian Institute of Science, Bangalore 560012 \\ Steven I. Marcus \\ Electrical Engineering Department \\ University of Maryland \\ College Park, Maryland 20742
}

\section{InTRODUCTION}

There is considerable literature on the Harnack inequality for uniformly elliptic partial differential equations [2], [3], [5]. Harnack's inequality, apart from being interesting in its own right, plays a very important role in the theory of partial differential equations. For example, it is applied to derive the interior estimates of the gradients of the solutions. Let us first state this result in the simplest situation. Let $\Omega$ be a bounded domain in $\mathbb{R}^{d}, \Gamma$

1991 Mathematics Subject Classification. Primary 35J45, Secondary 35J55, 35J65.

Key words and phrases. Harnack's inequality, elliptic systems. 
a closed subset of $\Omega$ and $u: \Omega \rightarrow \mathbb{R}$ a nonnegative harmonic function, i.e., $\Delta u=0$ in $\Omega$. Then there exists a constant $C$ which depends only on the dimension $d$, on the diameter of $\Omega$, and on the distance between $\Gamma$ and $\partial \Omega$, such that

$$
u(x) \leq C u(y), \quad \forall x, y \in \Gamma .
$$

The Harnack inequality is also valid for both weak and strong solutions of second-order, uniformly elliptic operators with bounded coefficients [2], [3]. Extensions to unbounded coefficients have also been established [9].

Consider a system of equations in $\boldsymbol{u}(x)=\left(u_{1}(x), \ldots, u_{n}(x)\right)$ of the form

$$
(L \boldsymbol{u})_{k}(x):=L_{k} u_{k}(x)+\sum_{\substack{j=1 \\ j \neq k}}^{n} c_{k j}(x) u_{j}(x)=0, \quad 1 \leq k \leq n,
$$

where $n$ is a positive integer and each $L_{k}$ is a second-order, uniformly elliptic operator given by

$$
L_{k}:=\sum_{i, j=1}^{d} a_{i j}^{k}(x) \frac{\partial^{2}}{\partial x_{i} \partial x_{j}}+\sum_{i=1}^{d} b_{i}^{k}(x) \frac{\partial}{\partial x_{i}}+c_{k k}(x) .
$$

The operator $L$ is called cooperative if the coupling coefficients $c_{k j}$ are nonnegative for $k \neq j$.

Definition 1.1. We denote by $\mathfrak{L}(\lambda, d, n)$ the class of all cooperative operators $L$ of the form (1.1)-(1.2), with coefficients $a_{i j}^{k} \in C^{0,1}\left(\mathbb{R}^{d}\right), b_{i}^{k}, c_{k j} \in$ $L^{\infty}\left(\mathbb{R}^{d}\right)$, bounded in $L^{\infty}$-norm by a constant $\lambda \geq 1$, and satisfying the uniform ellipticity condition

$$
\lambda^{-1}\|\zeta\|^{2} \leq \sum_{i, j=1}^{d} a_{i j}^{k}(x) \zeta_{i} \zeta_{j} \leq \lambda\|\zeta\|^{2}, \quad \text { for all } x, \zeta \in \mathbb{R}^{d}, 1 \leq k \leq n .
$$

A function $\boldsymbol{u}$ is called L-harmonic in a domain $\Omega \subset \mathbb{R}^{d}$ provided $\boldsymbol{u}$ is a strong solution of $L \boldsymbol{u}=0$ in the Sobolev space $W_{\ell \text { oc }}^{2, p}\left(\Omega ; \mathbb{R}^{n}\right)$, for some $p \in[1, \infty)$.

Systems of the above form appear in the study of jump diffusion processes with a discrete component [1]. In this paper, we obtain analogues 
of Harnack's inequality for $L$-harmonic functions of operators in the class $\mathfrak{L}(\lambda, d, n)$. We use the technique introduced by Krylov for estimating the oscillation of a harmonic function on bounded sets [3]. The main results are given in Section 2. Section 3 is devoted to proofs and auxiliary results.

After this work was submitted for publication, similar results were reported in [10]. Our work and [10] differ both in methodology and results. In [10] the authors assume Hölder continuous coefficients, and the proofs are based on estimates of the Green function in small balls, while this paper, motivated from a stochastic control problem, assumes only measurable coefficients, and the proofs are based on the approach of Krylov. Also, in our work an averaged coupling matrix (see Definition 2.1) appears explicitly in the Harnack constant. This enables us to provide a rather general version of the maximum principle and some further Harnack inequalities valid for a certain class of supersolutions.

\section{Main Results}

Throughout the paper, $\Omega$ denotes a bounded domain in $\mathbb{R}^{d}$. We first establish a weak version of Harnack's inequality, under general conditions.

Theorem 2.1. Let $\Gamma \subset \Omega$ be a closed set. There exists a constant $K_{1}>0$, depending only on $d, n$, the diameter of $\Omega$, the distance between $\Gamma$ and $\partial \Omega$, and the bound $\lambda$, such that for any nonnegative L-harmonic function $\boldsymbol{u}$ in $\Omega$, with $L \in \mathfrak{L}(\lambda, d, n)$,

$$
\sup _{x \in \Gamma}\left\{u_{i}(x)\right\} \leq K_{1} \max _{1 \leq k \leq n} \inf _{x \in \Gamma}\left\{u_{k}(x)\right\}, \quad \forall i \in\{1, \ldots, n\}
$$

An inequality stronger than (2.1) is obtained under an irreducibility condition on the coupling coefficients. We need to introduce some additional notation.

For a measurable set $A \subset \mathbb{R}^{d},|A|$ denotes the Lebesgue measure of $A$, while $\|\cdot\|_{p ; A}$ denotes the norm of $L^{p}(A), 1 \leq p \leq \infty$. Also, for $A \subset \Omega$, $\|\cdot\|_{k, p ; A}$ denotes the restriction to $A$ of the standard norm of $W^{k, p}(\Omega)$. 
These norms are extended to vector valued functions $\boldsymbol{u}$ using the convention $\|\boldsymbol{u}\|=\sum_{i=1}^{n}\left\|u_{i}\right\|$.

Definition 2.1. For $\Omega \subset \mathbb{R}^{d}$ and $L \in \mathfrak{L}(\lambda, d, n)$, let $\boldsymbol{C}_{L}(\Omega) \in \mathbb{R}^{n \times n}$ denote the matrix defined by

$$
\left[\boldsymbol{C}_{L}(\Omega)\right]_{i j}:=\frac{\left\|c_{i j}\right\|_{1 ; \Omega}}{|\Omega|}, \quad \text { for } i \neq j, \quad i, j \in\{1, \ldots, n\},
$$

with diagonal entries equal to 0 . Given a nonnegative matrix $M \in \mathbb{R}^{n \times n}$ and a pair $i, j \in\{1, \ldots, n\}$, we say that $j$ is reachable from $i$ provided that the $i j$ 'th element of $(I+M)^{n-1}$ is positive, and we denote this by $i \stackrel{M}{\longrightarrow} j$. Furthermore, the matrix $\boldsymbol{M}$ is called irreducible if $i \stackrel{M}{\longrightarrow} j$ for all $i, j \in$ $\{1, \ldots, n\}$; otherwise, it is called reducible. We say that $L \in \mathfrak{L}(\lambda, d, n)$ is $\mu_{\Omega^{-}}$ irreducible in $\Omega$ if there exists an irreducible matrix $\boldsymbol{S} \in \mathbb{R}^{n \times n}$, with elements in $\{0,1\}$ and $\mu_{\Omega} \in \mathbb{R}$, such that $\mu_{\Omega} \boldsymbol{C}_{L}(\Omega) \geq \boldsymbol{S}$ (here, the inequality is meant elementwise). The class of all $\mu_{\Omega}$-irreducible operators whose coefficients $a_{i j}^{k}$ have a uniform Lipschitz constant $\gamma$ is denoted by $\mathfrak{L}\left(\lambda, d, n, \gamma, \mu_{\Omega}\right)$.

Theorem 2.2. Let $\Gamma \subset \Omega$ be a closed set. There exists a positive constant $K_{2}=K_{2}\left(\Omega, \Gamma, \lambda, d, n, \gamma, \mu_{\Omega}\right)$ such that for any nonnegative L-harmonic function $\boldsymbol{u}$ in $\Omega$, with $L \in \mathfrak{L}\left(\lambda, d, n, \gamma, \mu_{\Omega}\right)$,

$$
u_{i}(x) \leq K_{2} u_{j}(y), \quad \forall x, y \in \Gamma, i, j \in\{1, \ldots, n\}
$$

More generally, if $L \in \mathfrak{L}(\lambda, d, n)$, and $\widetilde{c}_{\Omega}$ denotes the smallest positive element of $\boldsymbol{C}_{L}(\Omega)$, then

$$
u_{i}(x) \leq K_{2}^{\prime} u_{j}(y), \quad \forall x, y \in \Gamma, \text { if } j \stackrel{C_{L}(\Omega)}{\longrightarrow} i
$$

where $K_{2}^{\prime}=K_{2}\left(\Omega, \Gamma, \lambda, d, n, \gamma, \frac{1}{\widetilde{c}_{\Omega}}\right)$.

Remark 2.1. Let $\Gamma \subset \Omega$ and $L \in \mathfrak{L}(\lambda, d, n)$ be given. Then, for the existence of a constant $K_{2}>0$ satisfying (2.2) for all nonnegative $L$-harmonic functions $\boldsymbol{u}$ in $\Omega$, it is necessary that $L$ be $\mu_{\Omega}$-irreducible in $\Omega$. Otherwise, there exists a nontrivial partition $\left\{\mathcal{I}_{1}, \mathcal{I}_{2}\right\}$ of $\{1, \ldots, n\}$ such that $c_{i j}=0$ a.e. in $\Omega$, for 
all $(i, j) \in \mathcal{I}_{1} \times \mathcal{I}_{2}$; therefore, any nonzero $L$-harmonic function $\boldsymbol{u}$ satisfying $u_{k}=0$, for $k \in \mathcal{I}_{1}$, violates $(2.2)$.

There is a fair amount of work in the literature on maximum principles for cooperative, weakly-coupled systems [6], [7]. In [6], it is assumed that the coupling coefficients are positive. Note that the notion of irreducibility in Definition 2.1 is in an 'average' sense only and that $\boldsymbol{C}_{L}(\Omega)$ may be irreducible even if $\left[c_{i j}(x)\right]$ is reducible at every $x \in \Omega$. We state the following version of the strong maximum principle, which follows immediately from Theorem 2.2, and does not seem to be available in the existing literature.

Corollary 2.1. Let $L \in \mathfrak{L}(\lambda, d, n)$ be such that $\boldsymbol{C}_{L}(\Omega)$ is irreducible. Then any nonnegative L-harmonic function $\boldsymbol{u}$ in $\Omega$ is either positive in $\Omega$ or identically zero.

It is well known that, in general, there is no Harnack inequality for nonnegative $L$-superharmonic functions, i.e., functions $u$ satisfying $L u \leq 0$ in $\Omega$, for an elliptic operator $L$. Serrin [8] has utilized the maximum principle to provide a growth estimate in terms of the Harnack constant of a comparison function and the value of $\|L u\|_{\infty}$, but this estimate does not result in a Harnack inequality. Theorem 2.2 can be employed to provide a Harnack constant for all superharmonic functions $u$ for which $-L u$ lies in a convex positive cone of $L^{\infty}$. We introduce the following definition.

Definition 2.2. For a measurable set $A \subset \mathbb{R}^{d}$ having finite, nonzero measure and for a constant $\theta \geq 1$, we define the positive convex cone $\mathcal{K}(\theta, A) \subset$ $L^{\infty}\left(A ; \mathbb{R}^{n}\right)$ by

$$
\mathcal{K}(\theta, A):=\left\{\boldsymbol{f} \in L^{\infty}\left(A ; \mathbb{R}^{n}\right): \boldsymbol{f} \geq 0, \min _{1 \leq k \leq n} \frac{\left\|f_{k}\right\|_{1 ; A}}{|A|\left\|f_{k}\right\|_{\infty ; A}} \geq \frac{1}{\theta}\right\} .
$$

Suppose, for the moment, that $n=1$ and $u$ is a nonnegative function satisfying $L u=-f$ in $\Omega$, with $L \in \mathfrak{L}(\lambda, d, 1)$ and $f \in \mathcal{K}(\theta, \Omega)$. We form the cooperative system

$$
\begin{aligned}
L v_{1}+\frac{\theta f(x)}{\|f\|_{\infty ; \Omega}} v_{2} & =0 \\
\Delta v_{2} & =0 .
\end{aligned}
$$


Clearly, $\left(v_{1}, v_{2}\right)=\left(u, \theta^{-1}\|f\|_{\infty ; \Omega}\right)$ is a nonnegative solution and $\widetilde{c}_{\Omega} \geq 1$. Therefore, from (2.3), we deduce Harnack's inequality for $u$ by setting $\lambda=$ $\max \{\lambda, \theta\}$ and $\mu_{\Omega}=1$ in the Harnack constant $K_{2}$.

For the elliptic system in (1.1)-(1.2), this procedure leads to the following:

Corollary 2.2. Let $\Gamma \subset \Omega$ be a closed set and $\boldsymbol{u}$ a nonnegative function satisfying $-L \boldsymbol{u} \in \mathcal{K}(\theta, \Omega)$. The following are true:

(i) If $L \in \mathfrak{L}(\lambda, d, n, \gamma)$ then (2.1) holds, with a Harnack constant

$$
K_{1} K_{2}(\Omega, \Gamma, \max \{\lambda, \theta\}, d, 2 n, \gamma, 1)
$$

(ii) If $L \in \mathfrak{L}\left(\lambda, d, n, \gamma, \mu_{\Omega}\right)$, then (2.2) holds with a constant

$$
K_{2}\left(\Omega, \Gamma, \max \left\{\lambda, \frac{\theta}{\mu_{\Omega}}\right\}, d, 2 n, \gamma, \mu_{\Omega}\right)
$$

\section{Proofs of the Results}

If $\boldsymbol{u} \in W_{\ell \text { oc }}^{2, p}\left(\Omega ; \mathbb{R}^{n}\right)$, for some $p \in[1, \infty)$, is a solution of $L \boldsymbol{u}=\boldsymbol{f}$ in $\Omega$ and $\boldsymbol{f} \in L^{\infty}\left(\Omega ; \mathbb{R}^{n}\right)$, then $\boldsymbol{u} \in W_{\ell o c}^{2, p}\left(\Omega ; \mathbb{R}^{n}\right)$, for all $p \in[1, \infty)$. This fact follows from the interior $L^{p}$ estimates for second derivatives of uniformly elliptic equations and the well known Sobolev inequalities. However, the natural space for some considerations is $W^{2, d}$. This is the case, for example, for the Aleksandroff estimate (Lemma 3.2) and the comparison principle [2] which states that if $\boldsymbol{\varphi}, \boldsymbol{\psi} \in W_{\ell o c}^{2, d}\left(\Omega ; \mathbb{R}^{n}\right) \bigcap C^{0}\left(\bar{\Omega} ; \mathbb{R}^{n}\right)$ satisfy $L \boldsymbol{\varphi} \leq L \boldsymbol{\psi}$ in $\Omega$ and $\boldsymbol{\varphi} \geq \boldsymbol{\psi}$ on $\partial \Omega$, then $\boldsymbol{\varphi} \geq \boldsymbol{\psi}$ in $\bar{\Omega}$.

Let $\boldsymbol{u} \in W_{\ell \text { oc }}^{2, d}\left(\Omega ; \mathbb{R}^{n}\right)$ be a nonnegative solution of $L \boldsymbol{u}=0$ in $\Omega$, with $L \in$ $\mathfrak{L}(\lambda, d, n)$. Augmenting the dimension of the domain, let $I \subset \mathbb{R}$ be a bounded open interval and define the function $\boldsymbol{v}: \Omega \times I \rightarrow \mathbb{R}^{d}$ by $\boldsymbol{v}\left(x, x_{d+1}\right):=$ $\boldsymbol{u}(x) \exp \left(\sqrt{n \lambda} x_{d+1}\right)$, and the operator $\widetilde{L} \in \mathfrak{L}((n+1) \lambda, d+1, n)$ by

$$
\widetilde{L}_{k}:=L_{k}+\frac{\partial^{2}}{\partial x_{d+1}^{2}}-n \lambda+c_{k k}
$$


Then $\widetilde{L} \boldsymbol{v}=0$, and any Harnack estimates obtained for $\widetilde{L}$-harmonic functions clearly hold for $\boldsymbol{u}$. Observe that the coefficients $\widetilde{c}_{k j}$ of the operator $\widetilde{L}$ form a sub-stochastic matrix, i.e., they satisfy $\sum_{j=1}^{n} \widetilde{c}_{k j} \leq 0$, for all $k=1, \ldots, n$. Hence, without loss of generality, we restrict the proofs to operators in $\mathfrak{L}(\lambda, d, n)$ and $\mathfrak{L}\left(\lambda, d, n, \gamma, \mu_{\Omega}\right)$ whose coupling coefficients form a sub-stochastic matrix, and we denote the corresponding classes by $\mathfrak{L}_{\mathbf{0}}(\lambda, d, n)$ and $\mathfrak{L}_{\mathbf{0}}\left(\lambda, d, n, \gamma, \mu_{\Omega}\right)$, respectively.

Let $\mathfrak{U}_{\Omega}\left(\mathfrak{U}_{\Omega}^{-}\right)$denote the space of all nonnegative functions $\boldsymbol{u} \in$ $W_{\ell o c}^{2, d}\left(\Omega ; \mathbb{R}^{n}\right) \cap C^{0}\left(\bar{\Omega} ; \mathbb{R}^{n}\right)$, satisfying $L \boldsymbol{u}=0(L \boldsymbol{u} \leq 0)$ in $\Omega$, for some $L \in \mathfrak{L}_{\mathbf{0}}(\lambda, d, n)$. If $\xi \in \mathbb{R}$, then $\boldsymbol{u} \geq \xi$ is to be interpreted as $u_{i} \geq \xi$, for all $i \in\{1, \ldots, n\}$, and if $\boldsymbol{\xi}=\left(\xi_{1}, \ldots, \xi_{n}\right) \in \mathbb{R}^{n}$, then $\boldsymbol{u} \geq \boldsymbol{\xi} \Longleftrightarrow u_{i} \geq \xi_{i}$, for all $i \in\{1, \ldots, n\}$. In general, all scalar operations on $\mathbb{R}^{n}$-valued functions are meant to be componentwise. For greater clarity, we denote all $\mathbb{R}^{n}$-valued quantities by a bold letter. If $\Gamma$ is a closed subset of $\Omega, x \in \Omega$, and $\boldsymbol{\xi} \in \mathbb{R}_{+}^{n}$, we define

$$
\boldsymbol{\Psi}_{x}\left(\mathfrak{U}_{\Omega}, \Gamma ; \boldsymbol{\xi}\right):=\inf _{\boldsymbol{u} \in \mathfrak{U}_{\Omega}}\{\boldsymbol{u}(x): \boldsymbol{u} \geq \boldsymbol{\xi} \text { on } \Gamma\} .
$$

Lastly, deviating from the usual vector space notation, if $D$ is a cube in $\mathbb{R}^{d}$ and $\delta>0, \delta D$ denotes the cube which is concentric to $D$ and whose edges are $\delta$ times as long.

We start with a measure theoretic result, announced in [4]. For a proof see $[2]$.

Lemma 3.1. Let $K \subset \mathbb{R}^{d}$ be a cube, $\Gamma \subset K$ a closed subset, and $0<\alpha<1$. Define

$$
\begin{aligned}
\mathcal{Q} & :=\{Q: Q \text { is a subcube of } K \text { and }|Q \bigcap \Gamma| \geq \alpha|Q|\} \\
\widetilde{\Gamma} & :=\bigcup_{Q \in \mathcal{Q}}(3 Q \cap K) .
\end{aligned}
$$

Then either $\widetilde{\Gamma}=K$ or $|\widetilde{\Gamma}| \geq \frac{1}{\alpha}|\Gamma|$.

Next we state a variant of the weak maximum principle of A. D. Alexandroff. This particular form of the estimate is derived by first using a trans- 
formation to remove the first-order terms and then dominating the $L^{d}$ norm with the $L^{\infty}$ norm. The steps of the proof are quite standard and are therefore omitted.

Lemma 3.2. There exist constants $C_{1}>0$ and $\kappa_{0} \in(0,1]$ such that, if $D \subset \mathbb{R}^{d}$ is any cube of volume $|D| \leq \kappa_{0}$, and $\varphi \in W_{\text {loc }}^{2, d}(D) \cap C^{0}(\bar{D})$ satisfies $L_{k} \varphi \geq f$ in $D$, and $\varphi=0$ on $\partial D$, with $f \in L^{d}(D)$ and $L \in \mathfrak{L}(\lambda, d, n)$, then

$$
\sup _{x \in D}\{\varphi(x)\} \leq C_{1}|D|^{1 / d}\|f\|_{d ; D}
$$

For the remainder of this section, $D$ denotes an open cube in $\mathbb{R}^{d}$ of volume not exceeding the constant $\kappa_{0}$ in Lemma 3.2.

Lemma 3.3. There exist constants $\beta_{0}>0$ and $\alpha_{0}<1$ such that, if $\Gamma$ is a closed subset of some cube $D \subset \mathbb{R}^{d}$ satisfying $|\Gamma| \geq \alpha_{0}|D|$, then

$$
\inf _{x \in \frac{1}{3} D} \boldsymbol{\Psi}_{x}\left(\mathfrak{U}_{D}^{-}, \Gamma ; \boldsymbol{\xi}\right) \geq \beta_{0} \boldsymbol{\xi}, \quad \forall \boldsymbol{\xi} \in \mathbb{R}_{+}^{n}
$$

Proof. Observe that if $\boldsymbol{u} \in \mathfrak{U}_{D}^{-}$, then each component $u_{k}$ satisfies $L_{k} u_{k} \leq 0$ on $D$. Define $\varphi^{\prime}, \varphi^{\prime \prime} \in W_{\ell o c}^{2, d}(D) \cap C^{0}(\bar{D})$ by

$$
\begin{gathered}
L_{k} \varphi^{\prime}=-I_{\Gamma}, \quad L_{k} \varphi^{\prime \prime}=-I_{\Gamma^{c}}, \quad \text { in } D \\
\text { and } \quad \varphi^{\prime}=\varphi^{\prime \prime}=0, \quad \text { on } \partial D .
\end{gathered}
$$

Then $\varphi:=\varphi^{\prime}+\varphi^{\prime \prime}$ satisfies $L_{k} \varphi=-1$ in $D$ and $\varphi=0$ on $\partial D$. Without loss of generality, suppose $D$ is centered at the origin and consider the function

$$
\psi(x):=\prod_{i=1}^{d}\left(|D|^{2 / d}-4 x_{i}^{2}\right) .
$$

Clearly, $\psi=0$ on $\partial D$ and $\psi>0$ in $D$; moreover, there exists a positive constant $C_{2}$ such that

$$
\inf _{x \in \frac{1}{3} D}\{\psi(x)\} \geq C_{2}|D|^{2 / d}\left\|L_{k} \psi\right\|_{\infty ; D}, \quad \forall L \in \mathfrak{L}_{\mathbf{0}}(\lambda, d, n)
$$


Therefore, by the comparison principle

$$
\varphi(x) \geq \frac{\psi(x)}{\left\|L_{k} \psi\right\|_{\infty ; D}} \geq C_{2}|D|^{2 / d}, \quad \forall x \in \frac{1}{3} D .
$$

Using Lemma 3.2, we obtain

$$
\begin{aligned}
& \varphi^{\prime} \leq C_{1}|D|^{1 / d}|\Gamma|^{1 / d}=C_{1}|D|^{2 / d}\left(\frac{|\Gamma|}{|D|}\right)^{1 / d} \\
& \varphi^{\prime \prime} \leq C_{1}|D|^{1 / d}\left|\Gamma^{c}\right|^{1 / d}=C_{1}|D|^{2 / d}\left(1-\frac{|\Gamma|}{|D|}\right)^{1 / d} .
\end{aligned}
$$

By (3.1) and (3.2),

$$
\varphi^{\prime}(x) \geq C_{2}|D|^{2 / d}-C_{1}|D|^{2 / d}\left(1-\frac{|\Gamma|}{|D|}\right)^{1 / d}, \quad \forall x \in \frac{1}{3} D .
$$

On the other hand, since $L_{k} \varphi^{\prime}=0$ in $D \backslash \Gamma$ and $\varphi^{\prime}=0$ on $\partial D$, the comparison principle yields

$$
\inf _{x \in \frac{1}{3} D}\left\{u_{k}(x)\right\} \geq \xi_{k} \frac{C_{2}-C_{1}\left(1-\frac{|\Gamma|}{|D|}\right)^{1 / d}}{C_{1}\left(\frac{|\Gamma|}{|D|}\right)^{1 / d}} .
$$

Selecting $\alpha_{0}$ to satisfy

$$
\alpha_{0} \geq 1-\left(\frac{C_{2}}{2 C_{1}}\right)^{d}
$$

inequality (3.3) yields

$$
\inf _{x \in \frac{1}{3} D} u_{k}(x) \geq \frac{C_{2} \xi_{k}}{2 C_{1}} .
$$

Hence, the claim follows with $\beta_{0}=\frac{C_{2}}{2 C_{1}}$.

Lemma 3.4. For each $\delta>0$, there exists $k_{\delta}^{\prime}>0$ such that if $Q \subset(1-\delta) D$ is a subcube of an open cube $D \subset \mathbb{R}^{d}$, then

$$
\boldsymbol{\Psi}_{x}\left(\mathfrak{U}_{D}^{-}, \frac{1}{3} Q ; \boldsymbol{\xi}\right) \geq k_{\delta}^{\prime} \boldsymbol{\xi}, \quad \forall x \in 3 Q \bigcap(1-\delta) D, \quad \forall \boldsymbol{\xi} \in \mathbb{R}_{+}^{n}
$$


Proof. Let $B(r) \subset \mathbb{R}^{d}$ denote the ball of radius $r$ centered at the origin. We claim that there exists a constant $m_{0}>0$ such that if $r \leq 1$, then

$$
\inf _{x \in B\left(\frac{3 r}{4}\right)} \boldsymbol{\Psi}_{x}\left(\mathfrak{U}_{B(r)}^{-}, B\left(\frac{r}{4}\right) ; \boldsymbol{\xi}\right) \geq m_{0} \boldsymbol{\xi}, \quad \forall \boldsymbol{\xi} \in \mathbb{R}_{+}^{n}
$$

In order to establish (3.4) we use the function

$$
\varphi(x):=\exp \left\{16 \lambda^{2}(d+1)\left(1-\frac{\|x\|^{2}}{r^{2}}\right)\right\}-1, \quad x \in B(r)
$$

which satisfies $L_{k} \varphi(x) \geq 0$, for all $L \in \mathfrak{L}_{\mathbf{0}}(\lambda, d, n)$, provided $\|x\| \geq \frac{r}{4}$ and $r \leq 1$. By the comparison principle, (3.4) holds with

$$
m_{0}=\frac{e^{7 \lambda^{2}(d+1)}-1}{e^{15 \lambda^{2}(d+1)}-1}
$$

It follows that if $B(r)$ is centered at $y$, and $x$ is a point in $D$ such that the distance between $\partial D$ and the line segment joining $x$ and $y$ is at least $r$, then

$$
\boldsymbol{\Psi}_{x}\left(\mathfrak{U}_{D}^{-}, B\left(\frac{r}{4}\right) ; \boldsymbol{\xi}\right) \geq\left(m_{0}\right)^{\ell} \boldsymbol{\xi}, \quad \text { with } \ell=\left\lceil\frac{4\|x-y\|-r}{2 r}\right\rceil
$$

for all $\boldsymbol{\xi} \in \mathbb{R}_{+}^{n}$. If we define

$$
k_{\delta}^{\prime}:=m_{0}^{\ell(\delta)}, \quad \ell(\delta):=\left\lceil\frac{6 \sqrt{d}}{\min \{1, \delta\}}\right\rceil
$$

then an easy calculation, using (3.5) with $r=\min \left\{\frac{2}{3}, \frac{\delta}{2}\right\}|Q|^{1 / d}$, establishes the result.

Lemma 3.5. Suppose there exist constants $\varepsilon$ and $\theta$ such that if $\Gamma \subset(1-\delta) D$ is a closed subset of some cube $D$ and $\boldsymbol{\xi} \in \mathbb{R}_{+}^{n}$, then

$$
\inf _{x \in \frac{1}{3} D} \boldsymbol{\Psi}_{x}\left(\mathfrak{U}_{D}^{-}, \Gamma ; \boldsymbol{\xi}\right) \geq \varepsilon \boldsymbol{\xi}, \quad \text { whenever } \quad|\Gamma| \geq \theta|D|
$$

Then there exists a constant $k_{\delta}>0$ such that

$$
\inf _{x \in \frac{1}{3} D} \boldsymbol{\Psi}_{x}\left(\mathfrak{U}_{D}^{-}, \Gamma ; \boldsymbol{\xi}\right) \geq \varepsilon k_{\delta} \boldsymbol{\xi}, \quad \text { whenever } \quad|\Gamma| \geq \alpha_{0} \theta|D|
$$


where $\alpha_{0}$ is the constant in Lemma 3.3.

Proof. Suppose $|\Gamma| \geq \alpha_{0} \theta|D|$ and let $y \in \widetilde{\Gamma}$, with $\widetilde{\Gamma}$ as defined in Lemma 3.1 corresponding to $\alpha=\alpha_{0}$ and $K=(1-\delta) D$. Then there exists a subcube $Q \subset K$ such that $|\Gamma \bigcap Q| \geq \alpha_{0}|Q|$ and $y \in 3 Q \bigcap K$. We use the identities,

$$
\boldsymbol{\Psi}_{x}\left(\mathfrak{U}_{D}^{-}, \Gamma ; \boldsymbol{\xi}\right) \geq \boldsymbol{\Psi}_{x}\left(\mathfrak{U}_{D}^{-}, \widetilde{\Gamma} ; \inf _{y \in \widetilde{\Gamma}} \boldsymbol{\Psi}_{x}\left(\mathfrak{U}_{D}^{-}, \Gamma ; \boldsymbol{\xi}\right)\right)
$$

and

$$
\begin{aligned}
\boldsymbol{\Psi}_{y}\left(\mathfrak{U}_{D}^{-}, \Gamma ; \boldsymbol{\xi}\right) & \geq \boldsymbol{\Psi}_{y}\left(\mathfrak{U}_{D}^{-}, \frac{1}{3} Q ; \inf _{z \in \frac{1}{3} Q} \boldsymbol{\Psi}_{z}\left(\mathfrak{U}_{D}^{-}, \Gamma ; \boldsymbol{\xi}\right)\right) \\
& \geq \boldsymbol{\Psi}_{y}\left(\mathfrak{U}_{D}^{-}, \frac{1}{3} Q ; \inf _{z \in \frac{1}{3} Q} \boldsymbol{\Psi}_{z}\left(\mathfrak{U}_{Q}^{-}, \Gamma \bigcap Q ; \boldsymbol{\xi}\right)\right)
\end{aligned}
$$

By Lemma 3.3,

$$
\inf _{z \in \frac{1}{3} Q} \boldsymbol{\Psi}_{z}\left(\mathfrak{U}_{Q}^{-}, \Gamma \bigcap Q ; \boldsymbol{\xi}\right) \geq \beta_{0} \boldsymbol{\xi}
$$

while from Lemma 3.4 , we obtain $\boldsymbol{\Psi}_{y}\left(\mathfrak{U}_{D}^{-}, \frac{1}{3} Q ; \beta_{0} \boldsymbol{\xi}\right) \geq \beta_{0} k_{\delta}^{\prime} \boldsymbol{\xi}$, for all $y \in$ $3 Q \bigcap K$. Hence, combining (3.7) and (3.8), we obtain

$$
\inf _{y \in \widetilde{\Gamma}} \boldsymbol{\Psi}_{y}\left(\mathfrak{U}_{D}^{-}, \Gamma ; \boldsymbol{\xi}\right) \geq k_{\delta} \boldsymbol{\xi}, \quad \text { with } \quad k_{\delta}:=\beta_{0} k_{\delta}^{\prime}
$$

By Lemma 3.1, $|\widetilde{\Gamma}| \geq \frac{1}{\alpha_{0}}|\Gamma| \geq \theta|D|$. Therefore, by hypothesis,

$$
\inf _{x \in \frac{1}{3} D} \boldsymbol{\Psi}_{x}\left(\mathfrak{U}_{D}^{-}, \widetilde{\Gamma} ; k_{\delta} \boldsymbol{\xi}\right) \geq \varepsilon k_{\delta} \boldsymbol{\xi}
$$

which along with (3.6) and (3.9) yield the desired result.

Proposition 3.1. The following estimates hold:

(i) Let $D$ be a cube and $\Gamma \subset(1-\delta) D$ a closed subset. Then for all $\xi \in \mathbb{R}_{+}^{n}$,

$$
\inf _{x \in \frac{1}{3} D} \boldsymbol{\Psi}_{x}\left(\mathfrak{U}_{D}^{-}, \Gamma ; \boldsymbol{\xi}\right) \geq \beta_{0}\left(\frac{|\Gamma|}{|D|}\right)^{\rho(\delta)} \boldsymbol{\xi}, \quad \rho(\delta):=\frac{\log k_{\delta}}{\log \alpha_{0}}
$$


where the constants $\alpha_{0}, \beta_{0}$ and $k_{\delta}$ are as in Lemma 3.3 and Lemma 3.5.

(ii) There exists a real function $F$ defined on $[0,1]$, satisfying $F(\theta)>0$ for $\theta>0$, such that if $\Gamma \subset D$ is a closed subset of a cube $D$, then

$$
\inf _{x \in \frac{1}{3} D} \boldsymbol{\Psi}_{x}\left(\mathfrak{U}_{D}^{-}, \Gamma ; \boldsymbol{\xi}\right) \geq F\left(\frac{|\Gamma|}{|D|}\right) \boldsymbol{\xi}, \quad \forall \boldsymbol{\xi} \in \mathbb{R}_{+}^{n}
$$

Proof. Part (i) is direct consequence of Lemmas 3.3 and 3.5. For part (ii), choose $\delta=\frac{|\Gamma|}{4 d|D|}$. Then,

$$
\frac{|\Gamma \bigcap(1-\delta) D|}{|D|} \geq \frac{|\Gamma|}{|D|}-\left(1-(1-\delta)^{d}\right) \geq \frac{|\Gamma|}{|D|}-d \delta \geq \frac{3|\Gamma|}{4|D|} .
$$

Since

$$
\boldsymbol{\Psi}_{x}\left(\mathfrak{U}_{D}^{-}, \Gamma ; \boldsymbol{\xi}\right) \geq \boldsymbol{\Psi}_{x}\left(\mathfrak{U}_{D}^{-}, \Gamma \bigcap(1-\delta) D ; \boldsymbol{\xi}\right)
$$

then if we let

$$
F(\theta):=\beta_{0}\left(\frac{3 \theta}{4}\right)^{\rho\left(\frac{\theta}{4 d}\right)},
$$

the bound in (3.11) follows from (3.10) and (3.12).

Proposition 3.2. If $D$ is a cube, $\boldsymbol{u} \in \mathfrak{U}_{D}$ and $q=F\left(\frac{1}{2}\right)$, with $F(\cdot)$ as defined in Proposition 3.1 (ii), then

$$
\operatorname{osc}\left(u_{k} ; \frac{1}{3} D\right) \leq\left(1-\frac{q}{2}\right) \max _{1 \leq k \leq n} \sup _{x \in D}\left\{u_{k}(x)\right\}, \quad \forall k \in\{1, \ldots, n\}
$$

where $\operatorname{osc}(f ; A)$ denotes the oscillation of a function $f$ over a set $A$.

Proof. Let

$$
\begin{array}{rlrl}
M_{k}^{a} & :=\sup _{x \in \frac{1}{3} D}\left\{u_{k}(x)\right\}, & M^{a}:=\max _{1 \leq k \leq n} M_{k}^{a} \\
m_{k}^{a}:=\inf _{x \in \frac{1}{3} D}\left\{u_{k}(x)\right\}, & m^{a}:=\min _{1 \leq k \leq n} m_{k}^{a}
\end{array}
$$

and $M^{b}, m^{b}$ be the corresponding bounds relative to $D$. Consider the sets

$$
\begin{aligned}
& \Gamma_{1}^{(k)}:=\left\{x \in D: u_{k}(x) \leq \frac{M^{b}+m^{b}}{2}\right\} \\
& \Gamma_{2}^{(k)}:=\left\{x \in D: u_{k}(x) \geq \frac{M^{b}+m^{b}}{2}\right\} .
\end{aligned}
$$


Suppose $\left|\Gamma_{1}^{(k)}\right| \geq \frac{1}{2}|D|$. Since $M^{b}-\boldsymbol{u}$ is nonnegative and $M^{b}-u_{k} \geq \frac{M^{b}-m^{b}}{2}$ in $\Gamma_{1}^{(k)}$, applying Proposition 3.1 (ii), we get

$$
M^{b}-u_{k}(x) \geq q \frac{M^{b}-m^{b}}{2}, \quad \forall x \in \frac{1}{3} D .
$$

Consequently, $M_{k}^{a} \leq M^{b}-q \frac{M^{b}-m^{b}}{2}$, and since $m^{a} \geq m^{b}$, we obtain

$$
M_{k}^{a}-m^{a} \leq M^{b}-m^{b}-q \frac{M^{b}-m^{b}}{2} \leq\left(1-\frac{q}{2}\right) M^{b} .
$$

On the other hand, if $\left|\Gamma_{2}^{(k)}\right| \geq \frac{1}{2}|D|$, the analogous argument relative to the nonnegative function $\boldsymbol{u}$, yields

$$
M^{a}-m_{k}^{a} \leq\left(1-\frac{q}{2}\right) M^{b}
$$

and the result follows by (3.13)-(3.14).

Proposition 3.3. There exists a constant $M_{1}>0$ such that, for any $\boldsymbol{u} \in$ $\mathfrak{U}_{D}$

$$
\sup _{x \in \frac{1}{9} D}\left\{u_{i}(x)\right\} \leq M_{1} \max _{1 \leq k \leq n} \inf _{x \in \frac{1}{9} D}\left\{u_{k}(x)\right\}, \quad \forall i \in\{1, \ldots, n\}
$$

Proof. Let $\beta_{0}$ be as in Lemma 3.3 and $\rho(\cdot)$ and $q$ as in (3.10) and Proposition 3.2 , respectively. Define

$$
\rho:=\frac{1}{d \rho\left(\frac{2}{3}\right)} \quad \text { and } \quad q_{0}:=\frac{\left(1-\frac{q}{4}\right)}{\left(1-\frac{q}{2}\right)} .
$$

We claim that the value of the constant $M_{1}$ may be chosen as

$$
M_{1}:=\frac{4 q_{0}}{q \beta_{0}}\left[\frac{27 n^{1 / d}}{2\left(q_{0}^{\rho}-1\right)}\right]^{1 / \rho} .
$$

We argue by contradiction. Suppose $\boldsymbol{u}$ violates this bound and let $\left\{x^{(1)}, \ldots, x^{(n)}\right\}$ denote the points in $\frac{1}{9} \bar{D}$ where the minima of $\boldsymbol{u}$ are attained, i.e.,

$$
\inf _{x \in \frac{1}{9} D}\left\{u_{k}(x)\right\}=u_{k}\left(x^{(k)}\right), \quad 1 \leq k \leq n
$$


Without loss of generality, suppose that $\max _{1 \leq k \leq n}\left\{u_{k}\left(x^{(k)}\right)\right\}=1$ and that for some $y_{0} \in \frac{1}{9} D$ and $k_{0} \in\{1, \ldots, n\}, u_{k_{0}}\left(y_{0}\right)=M>a M_{1}$, with $a>1$. Using the estimate for the growth of the oscillation of $\boldsymbol{u}$ in Proposition 3.2, we then show that $\boldsymbol{u}$ has to be unbounded in $\frac{1}{3} D$. By hypothesis, $\frac{M}{a}$ exceeds $M_{1}$ in (3.16) and, in order to facilitate the construction which follows, we choose to express this as

$$
\frac{1}{9}+3 n^{1 / d}\left(\frac{4 a}{q \beta_{0} M}\right)^{\rho} \sum_{i=0}^{\infty}\left(\frac{1}{q_{0}}\right)^{i \rho}<\frac{1}{3} .
$$

For $\xi>0$, define

$$
\mathcal{D}_{k}^{(\xi)}:=\left\{x \in \frac{1}{3} \bar{D}: u_{k}(x) \geq \xi\right\}, \quad \mathcal{D}^{(\xi)}:=\bigcup_{1 \leq k \leq n} \mathcal{D}_{k}^{(\xi)} .
$$

If $\mathbf{1}_{k} \in \mathbb{R}_{+}^{n}$ stands for the vector whose $k$-th component is equal to 1 and the others 0 , then

$$
\boldsymbol{u}\left(x^{(k)}\right) \geq \boldsymbol{\Psi}_{x^{(k)}}\left(\mathfrak{U}_{D}, \mathcal{D}_{k}^{(\xi)} ; \xi \mathbf{1}_{k}\right), \quad \forall k \in\{1, \ldots, n\}
$$

while, on the other hand, Proposition 3.1 yields

$$
\boldsymbol{\Psi}_{x^{(k)}}\left(\mathfrak{U}_{D}, \mathcal{D}_{k}^{(\xi)} ; \xi \mathbf{1}_{k}\right) \geq \beta_{0}\left(\frac{\left|\mathcal{D}_{k}^{(\xi)}\right|}{|D|}\right)^{\rho\left(\frac{2}{3}\right)} \xi \mathbf{1}_{k}, \quad \forall k \in\{1, \ldots, n\} .
$$

By (3.18)-(3.19) and using (3.15), we obtain the estimate

$$
\left|\mathcal{D}^{(\xi)}\right| \leq \sum_{1 \leq k \leq n}\left|\mathcal{D}_{k}^{(\xi)}\right| \leq \sum_{1 \leq k \leq n}\left(\frac{u_{k}\left(x^{(k)}\right)}{\xi \beta_{0}}\right)^{\rho d}|D| \leq n\left(\frac{1}{\xi \beta_{0}}\right)^{\rho d}|D|,
$$

for all $\xi>0$. Choosing $\xi=\frac{q M}{4}$, we have by (3.20)

$$
\left|\left\{x \in \frac{1}{3} D: \max _{1 \leq k \leq n}\left\{u_{k}(x)\right\} \geq \frac{q M}{4}\right\}\right| \leq n\left(\frac{4}{q \beta_{0} M}\right)^{\rho d}|D| .
$$

Hence, if $Q_{0}$ is a cube of volume $\left|Q_{0}\right|=n\left(\frac{4 a}{q \beta_{0} M}\right)^{\rho d}|D|$ centered at $y_{0}$, then

$$
\operatorname{osc}\left(u_{k_{0}} ; Q_{0}\right)>\left(1-\frac{q}{4}\right) M
$$


By Proposition 3.2 and $(3.21)$, there exists $y^{(1)} \in 3 Q_{0}$ and $k_{1} \in\{1, \ldots, n\}$ such that

$$
u_{k_{1}}\left(y^{(1)}\right)>\frac{\left(1-\frac{q}{4}\right)}{\left(1-\frac{q}{2}\right)} M=q_{0} M .
$$

Note that (3.17) implies that $3 Q_{0} \subset \frac{1}{3} D$. This allows us to repeat the argument above, this time choosing $\xi=q_{0} \frac{q M}{4}$ in (3.20) and a cube $Q_{1}$ of volume $n\left(\frac{4 a}{q_{0} q \beta_{0} M}\right)^{\rho d}|D|$ centered at $y^{(1)}$, to conclude that there exists $y^{(2)} \in 3 Q_{1}$ and $k_{2} \in\{1, \ldots, n\}$ such that $u_{k_{2}}\left(y^{(2)}\right) \geq q_{0}^{2} M$. Inductively, we construct a sequence $\left\{y^{(i)}, k_{i}, Q_{i}\right\}_{i=0}^{\infty}$ satisfying, for all $i=0,1, \ldots$,

$$
\begin{gathered}
y^{(0)}=y_{0} \in \frac{1}{9} \bar{D} \cap Q_{0}, \quad y^{(i)} \in Q_{i} \bigcap 3 Q_{i-1}, \\
\left|Q_{i}\right|^{1 / d}=n^{1 / d}\left(\frac{1}{q_{0}}\right)^{i \rho}\left(\frac{4 a}{q \beta_{0} M}\right)^{\rho}|D|^{1 / d}, \\
u_{k_{i}}\left(y^{(i)}\right) \geq q_{0}^{i} M .
\end{gathered}
$$

The inequality in (3.17) guarantees that $y^{(i)} \in \frac{1}{3} D$, for all $i$. Hence, (3.22) implies that $\boldsymbol{u}$ is unbounded in $\frac{1}{3} D$, and we reach a contradiction.

Theorem 2.1 now follows via the standard technique of selecting an appropriate cover of $\Gamma$ consisting of congruent cubes and applying the estimates in Proposition 3.1 and Proposition 3.3.

We next proceed to prove Theorem 2.2. We need the following lemma.

Lemma 3.6. Let $D \subset \mathbb{R}^{d}$ be a cube, $L \in \mathfrak{L}_{\mathbf{0}}(\lambda, d, 1, \gamma)$, and $f \in \mathcal{K}(\theta, D)$. There exists a constant $C^{\prime}=C^{\prime}(|D|, \lambda, d, \gamma, \theta)>0$ such that if $\varphi$ is a solution to the Dirichlet problem $L \varphi=-f$ on $D$, with $\varphi=0$ on $\partial D$, then

$$
\inf _{x \in \frac{1}{3} D}\{\varphi(x)\} \geq C^{\prime}\|f\|_{\infty ; D}
$$

Proof. First note that the Dirichlet problem has a unique strong solution $\varphi \in W_{\ell o c}^{2, p}(D) \bigcap C^{0}(\bar{D})$, for all $p \in[d, \infty)$. Then, arguing by contradiction, suppose there exists a sequence of operators $\left\{L^{(m)}\right\}_{m=1}^{\infty} \subset$ $\mathfrak{L}_{\mathbf{0}}(\lambda, d, 1, \gamma)$, with coefficients $\left\{a_{i j}^{(m)}, b_{i}^{(m)}, c^{(m)}\right\}$, and a sequence of functions 
$\left\{f^{(m)}\right\}_{m=1}^{\infty} \subset \mathcal{K}(\theta, D)$, with $\left\|f^{(m)}\right\|_{\infty ; D}=1$, such that the corresponding solutions $\left\{\varphi^{(m)}\right\}_{m=1}^{\infty}$ satisfy

$$
\inf _{x \in \frac{1}{3} D}\left\{\varphi^{(m)}(x)\right\}<\frac{1}{m^{2}}, \quad m=1,2, \ldots
$$

By Proposition 3.1,

$$
\left|\left\{x \in(1-\delta) D: \varphi^{(m)}(x) \geq \frac{1}{m}\right\}\right| \leq\left(\frac{1}{\beta_{0} m}\right)^{1 / \rho(\delta)}|D|, \quad \forall \delta>0 .
$$

Since the sequence $\varphi^{(m)}$ is bounded in $L^{\infty}(D)$ (by Lemma 3.2), it follows from (3.23) that $\varphi^{(m)} \rightarrow 0$ in $L^{p}(D)$, as $m \rightarrow \infty$, for all $p \in[1, \infty)$. For any subcube $D^{\prime}=\delta D$, with $\delta<1$, and $p \in[1, \infty)$, we use the well known estimate

$$
\left\|\varphi^{(m)}\right\|_{2, p ; D^{\prime}} \leq C^{\prime \prime}\left(\left\|\varphi^{(m)}\right\|_{p ; D}+\left\|f^{(m)}\right\|_{p ; D}\right)
$$

for some constant $C^{\prime \prime}=C^{\prime \prime}(|D|, p, \delta, \lambda, d, \gamma)$, to conclude that the first and second derivatives of $\varphi^{(m)}$ converge weakly to 0 in $L^{p}\left(D^{\prime}\right)$, for all $p \in[1, \infty)$. In turn, since $W_{0}^{2, p}\left(D^{\prime}\right) \hookrightarrow W_{0}^{1, p}\left(D^{\prime}\right)$ is compact for $p>d$, using the standard approximation argument, we deduce that $\frac{\partial \varphi^{(m)}}{\partial x_{i}}$ converges in $L^{p}\left(D^{\prime}\right)$ strongly, for all $i=1, \ldots, d$. Also, since the sequence $\left\{a_{i j}^{(m)}\right\}$ is uniformly Lipschitz, we can extract a subsequence which converges uniformly. The previous arguments combined imply that $\left\{L^{(m)} \varphi^{(m)}\right\}$ converges weakly to 0 in $L^{p}\left(D^{\prime}\right), p \in[1, \infty)$. On the other hand, if we choose $\delta \geq\left(1-\frac{1}{2 \theta}\right)^{1 / d}$, an easy calculation yields,

$$
\int_{D^{\prime}} f^{(m)}(x) d x \geq \frac{|D|}{2 \theta}, \quad m=1,2, \ldots,
$$

resulting in a contradiction.

Proof of Theorem 2.2. Let $L \in \mathfrak{L}_{\mathbf{0}}\left(\lambda, d, n, \gamma, \mu_{\Omega}\right)$ and $\boldsymbol{S}=\left[s_{i j}\right]$ as in Definition 2.1. Select a collection $\left\{D_{\ell}, \ell=1, \ldots, \ell_{0}\right\}$ of disjoint, congruent open cubes, whose closures form a cover of $\Gamma$, in such a manner that $3 \bar{D}_{\ell} \subset \Omega, 1 \leq \ell \leq \ell_{0}$, and $\mathcal{D}:=\bigcup_{\ell=1}^{\ell_{0}} \bar{D}_{\ell}$ is a connected set satisfying 
$|\mathcal{D}| \leq\left(1-\frac{1}{2 \lambda \mu_{\Omega}}\right)|\Omega|$. It follows that $2 \mu_{\Omega} \boldsymbol{C}_{L}(\mathcal{D}) \geq \boldsymbol{S}$. Therefore, for each pair $i, j \in\{1, \ldots, n\}, i \neq j$, there exists $\ell(i, j) \in\left\{1, \ldots, \ell_{0}\right\}$ such that

$$
\left\|c_{i j}\right\|_{\infty ; D_{\ell(i, j)}} \geq \frac{\left\|c_{i j}\right\|_{1 ; D_{\ell(i, j)}}}{\left|D_{\ell(i, j)}\right|} \geq \frac{s_{i j}}{2 \mu_{\Omega}} .
$$

Define a collection $\left\{\varphi_{i j}\right\}_{i \neq j} \subset W_{\ell o c}^{2, d}\left(D_{\ell(i, j)}\right) \cap C^{0}\left(\bar{D}_{\ell(i, j)}\right)$, by

$$
L_{i} \varphi_{i j}=-c_{i j} \quad \text { in } D_{\ell(i, j)}, \quad \text { and } \quad \varphi_{i j}=0 \quad \text { on } \partial D_{\ell(i, j)} \text {. }
$$

Then, by Lemma 3.6 and (3.24), there exists a constant $C^{\prime}>0$, such that

$$
\varphi_{i j}(x) \geq \frac{C^{\prime}}{2 \mu_{\Omega}} s_{i j}, \quad \forall x \in \frac{1}{3} D_{\ell(i, j)}, i \neq j .
$$

By the comparison principle,

$$
u_{i}(x) \geq \varphi_{i j}(x) \inf _{z \in D_{\ell(i, j)}}\left\{u_{j}(z)\right\}, \quad \forall x \in D_{\ell(i, j)}, i \neq j .
$$

By Proposition 3.1, (3.25) and (3.26),

$$
\begin{aligned}
u_{i}(y) & \geq F\left(\frac{1}{9^{d}}\right) \inf _{x \in \frac{1}{3} D_{\ell(i, j)}}\left\{u_{i}(x)\right\} \\
& \geq \varepsilon^{\prime} F\left(\frac{1}{9^{d}}\right) s_{i j} \inf _{z \in D_{\ell(i, j)}}\left\{u_{j}(z)\right\}, \quad \forall y \in D_{\ell(i, j)}, i \neq j .
\end{aligned}
$$

Moreover, provided $3 D_{k} \supset D_{k^{\prime}}, 1 \leq k, k^{\prime} \leq \ell_{0}$, Proposition 3.1 also asserts that

$$
\inf _{x \in D_{k}}\{\boldsymbol{u}(x)\} \geq F\left(\frac{1}{3^{d}}\right) \inf _{x \in D_{k^{\prime}}}\{\boldsymbol{u}(x)\},
$$

from which we deduce that

$$
\inf _{x \in \mathcal{D}_{k}}\{\boldsymbol{u}(x)\} \geq\left(F\left(\frac{1}{3^{d}}\right)\right)^{\ell_{0}} \inf _{x \in D_{\ell}}\{\boldsymbol{u}(x)\}, \quad \forall k, \ell \in\left\{1, \ldots, \ell_{0}\right\} .
$$

Therefore, by (3.27) and (3.28), for all $i \neq j$,

$$
\begin{aligned}
\inf _{x \in \mathcal{D}}\left\{u_{i}(x)\right\} & \geq\left(F\left(\frac{1}{3^{d}}\right)\right)^{\ell_{0}} \inf _{y \in D_{\ell(i, j)}}\left\{u_{i}(y)\right\} \\
& \geq \frac{C^{\prime}}{2 \mu_{\Omega}} F\left(\frac{1}{9^{d}}\right)\left(F\left(\frac{1}{3^{d}}\right)\right)^{\ell_{0}} s_{i j} \inf _{z \in D_{\ell(i, j)}}\left\{u_{j}(z)\right\} \\
& \geq \frac{C^{\prime}}{2 \mu_{\Omega}} F\left(\frac{1}{9^{d}}\right)\left(F\left(\frac{1}{3^{d}}\right)\right)^{2 \ell_{0}} s_{i j} \inf _{x \in \mathcal{D}}\left\{u_{j}(x)\right\},
\end{aligned}
$$


and in turn, the irreducibility of $S$ implies that, for all $i, j \in\{1, \ldots, n\}$,

$$
\inf _{x \in \mathcal{D}}\left\{u_{i}(x)\right\} \geq\left(\frac{C^{\prime}}{2 \mu_{\Omega}} F\left(\frac{1}{9^{d}}\right)\left(F\left(\frac{1}{3^{d}}\right)\right)^{2 \ell_{0}}\right)^{n-1} \inf _{x \in \mathcal{D}}\left\{u_{j}(x)\right\} .
$$

The result follows by combining (3.29) and the estimate in Theorem 2.1 relative to the closed set $\mathcal{D} \subset \Omega$.

\section{ACKOWLEDGEMENTS}

The authors wish to thank Prof. S.R.S. Varadhan for explaining the work of Krylov and Safonov on Harnack's inequality. The proof of Theorem 2.1 is inspired by his notes on the proof of Harnack's inequality for a uniformly

elliptic operator. Part of this work was completed while the first author was visiting the Department of Mathematics at the Indian Institute of Science in Bangalore. Their kind hospitality is deeply appreciated.

\section{REFERENCES}

1. M. K. Ghosh, A. Arapostathis and S. I. Marcus, Optimal control of switching diffusions with application to flexible manufacturing systems, SIAM J. Control Optim. 31 (1993), 1183-1204.

2. D. Gilbarg and N. Trudinger, Elliptic Partial Differential Equations of Second Order, Springer-Verlag, Berlin, 1985.

3. N. V. Krylov, Nonlinear Elliptic and Parabolic Equations of the Second Order, D. Reidel Publishing Co., Dordrecht, 1987.

4. N. V. Krylov and M. V. Safonov, An estimate of the probability that a diffusion process hits a set of positive measure, Soviet Math. Dokl. 20 (1979), 253-255.

5. O. A. Ladyzhenskaya and N. N. Ural'ceva, Linear and Quasilinear Elliptic Equations, Academic Press, New York, 1968.

6. J. López-Gómez and M. Molina-Meyer, The maximum principle for cooperative weakly coupled elliptic systems and some applications, Differential and Integral Equations $\mathbf{7}$ (1994), 383-398.

7. M. H. Protter and H. F. Weinberger, Maximum Principles in Differential Equations, Prentice-Hall, Englewood Cliffs, New Jersey, 1967.

8. J. B. Serrin, On the Harnack inequality for linear elliptic equations, J. d' Analyse Math. 4 (1954-56), 292-308.

9. K.-T. Sturm, Harnack's inequality for parabolic operators with singular low order terms, Math. Z. 216 (1994), 593-611.

10. Z.-Q. Chen and Z. Zhao, Harnack principle for weakly coupled elliptic systems, Journal of Differential Equation 139 (1997), 261-282. 